\title{
Selection for Liver Transplantation: Indications and Evaluation
}

\author{
Nadim Mahmud ${ }^{1}$ (i) \\ Published online: 19 June 2020 \\ (C) Springer Science+Business Media, LLC, part of Springer Nature 2020
}

\begin{abstract}
Purpose of Review Liver transplantation is an important therapeutic option for patients with life-limiting liver disease, which may present in the form of acute liver failure, end-stage chronic liver disease, primary hepatic cancers, or inborn metabolic disorders. While significant strides have been made with respect to liver transplantation outcomes, the practice is constrained by an organ supply/demand mismatch. The purpose of this review, therefore, is to review the general indications and contraindication to liver transplantation, and to provide an overview of the transplant evaluation process. These considerations ultimately shape the specific criteria for patient selection, which will continue to evolve as means are developed to expand the donor pool, improve surgical techniques, broaden indications for safe transplant, and extend the lifetime of a graft.

Recent Findings Selected patients with unresectable hilar cholangiocarcinoma may be candidates for liver transplantation. Patients over 65 years may be transplant candidates if they possess a favorable comorbidity profile. Patients at body mass index extremes $(\geq 40$ or $<18.5)$ have increased post-transplant mortality and require nutritional evaluation.

Summary Liver transplantation may be life saving for patients with acute liver failure or end-stage liver disease. It is therefore critical for healthcare providers caring for patients with liver disease to be familiar with the general indications for transplantation and to know when it is appropriate or inappropriate to refer for transplant evaluation.
\end{abstract}

Keywords Liver transplantation - Cirrhosis · Acute liver failure · Model for end-stage liver disease (MELD) · Hepatocellular carcinoma $\cdot$ Cholangiocarcinoma

\section{Introduction}

Liver transplantation (LT) may be curative or life prolonging for appropriately selected patients with acute liver failure, advanced cirrhosis, hepatic malignancy, or inborn metabolic disorders. Given vast improvements in surgical technique, organ preservation and procurement, and immunosuppression over the past several decades, modern LT is characterized by remarkable improvements in post-transplant patient survival, graft survival, and quality of life. In 2010, Duffy et al. demonstrated an actuarial 20-year post-transplant survival of $52 \%$ for the patient and $42 \%$ for the graft. They also reported

This article is part of the Topical Collection on Management of the Cirrhotic Patient

Nadim Mahmud

nadim@pennmedicine.upenn.edu

1 Division of Gastroenterology, Hospital of the University of Pennsylvania, Philadelphia, PA, USA improved health-related quality of life in 20-year survivors as compared with control patients with chronic liver disease, diabetes, or congestive heart failure [1]. Taken together, LT is now regarded to be a durable surgery and is the therapy of choice for a broad range of conditions that severely impair hepatic function.

In the face of excellent LT outcomes, a principal challenge is the current burden of severe liver disease and the associated scarcity of donor organs. More than 6000 liver transplants have been performed on an annual basis in the United States (US) since 2004, with 7841 performed in 2016 alone [2••]. However, there are currently in excess of 14,000 patients registered on the transplant waiting list at any given time, and more than 3000 either die or are delisted annually as a result of worsening illness that precludes transplant. This supplydemand mismatch places emphasis on an appropriate patient selection system that balances principles of benefit, justice, and fairness. Although the approach to transplantation varies across the globe, this review will focus on the US allocation system and its attendant approach to patient selection and pretransplant evaluation. 


\section{The US Liver Transplant Allocation System}

In 1984, the National Organ Transplant Act established the Organ Procurement and Transplant Network (OPTN), a national system for curating and developing policy around waiting lists, organ donation, matching, and transplantation. The United Network for Organ Sharing (UNOS) is a federally funded organization that currently manages the OPTN. In contrast to other solid organ systems, the current allocation of deceased donor liver grafts is based purely on waitlist mortality, where patients with the highest short-term mortality will be offered donor organs first. Patients on the LT list are prioritized based on their Model for End-Stage Liver Diseasesodium (MELD-Na) score, which is calculated from bilirubin, international normalized ratio (INR), creatinine, and sodium. MELD-Na orders transplant candidates from less ill (MELD 6) to extremely ill (MELD 40); the 3-month mortalities of these extremes are $7 \%$ and $90 \%$, respectively.

There are several important exceptions to the standard MELD scoring system. First, a status 1 listing is reserved for patients with rapidly progressive liver disease and a prognosis of hours to days to live without transplantation, typically patients with acute liver failure or post-transplant graft failure. Status 1 escalates a patient to the top of the waiting list irrespective of MELD score and also broadens geographic access to potential donors. Second, there are a handful of identified circumstances in which the MELD score underestimates the true short-term mortality of a patient with liver disease. In these cases, MELD exception points can be granted provided specific criteria are met. Finally, if a patient is felt to have a risk of death that is not captured by either of the above mechanisms - usually for exceptionally rare hepatic disorders - a clinical team may submit an appeal for consideration of non-standard exception points.

\section{Indications for Liver Transplantation}

Three underlying principles dictate which patients should be referred for and potentially undergo transplant. First, the recipient should have irreversible liver disease that is expected to be fatal without transplantation. This disease may be acute or chronic in nature. Second, the patient should have sufficient reserve to survive the operative and perioperative period. Finally, the candidate should be expected to have significant survival and quality of life benefit from LT. The following section reviews the accepted indications for LT in the US and the associated clinical evidence. These indications are largely consistent with the most recent practice guidelines as detailed by the American Association for the Study of Liver Disease (AASLD; Table 1) $[3 \cdot \bullet]$.

\section{Acute Liver Failure}

A sudden insult with rapid loss of hepatic function may lead to acute liver failure (ALF), which is defined as coagulopathy (INR $\geq 1.5$ ), hepatic encephalopathy (HE), and onset less than 26 weeks in a patient without underlying chronic liver disease. Approximately 2000 cases of ALF occur in the US annually [4], with disproportionate involvement of younger patients (median age 38 ), female predominance (73\%), and a median time to death of 5 days without transplantation [5]. The most common cause of ALF is acetaminophen overdose (39\%), followed by undetermined etiology (17\%), idiosyncratic drug reaction (13\%), acute hepatitis A or B infection (12\%), and

Table 1 Indications and contraindications for liver transplant

\section{Indications}

Acute liver failure

Hepatic artery thrombosis within 14 days of liver transplant

Cirrhosis with:

Decompensation (variceal bleeding, hepatic encephalopathy, or ascites) MELD score $\geq 15$

Hepatopulmonary syndrome or portopulmonary hypertension (select patients)

Primary hepatic neoplasms:

Hilar cholangiocarcinoma (highly selected, after neoadjuvant therapy protocol)

Hepatocellular carcinoma within the Milan criteria

Inborn metabolic conditions:

Cystic fibrosis with concomitant lung and liver disease

Primary hyperoxaluria type I with significant renal insufficiency

Familial amyloid polyneuropathy

\section{Contraindications}

Absolute contraindications

Active extrahepatic malignancy

Intrahepatic cholangiocarcinoma

Hepatocellular carcinoma outside Milan criteria or metastatic

Severe cardiopulmonary disease

Uncontrolled sepsis

Active alcohol or illicit substance abuse

Acquired immune deficiency syndrome (AIDS)

Persistent non-compliance or lack of social support

Technical and/or anatomic barriers to liver transplantation

Relative contraindications

Advanced age

Portal venous thrombosis

Human immunodeficiency virus (HIV) infection

MELD score $<15$

Morbid obesity (body mass index [BMI] $\geq 40$ )

Poor medical compliance or social support

Active psychiatric comorbidities 
shock $(6 \%)$. The proportion of ALF caused by acetaminophen overdose in the US has increased over time, with more recent estimates in excess of $50 \%$ of cases [6].

Depending on the severity of injury, host factors, and medical resources available, there are three potential clinical courses for patients with ALF: (1) spontaneous recovery, (2) LT, or (3) rapid progression to multiorgan dysfunction and death. In a large series, $43 \%$ of ALF patients experienced spontaneous recovery, 29\% underwent LT, and $25 \%$ died either awaiting transplant or were never listed [5]. For any patient with ALF, expeditious referral to a transplant center is paramount. Because of the condition's high short-term mortality, patients listed for transplant are eligible for status 1a. Specific criteria for status 1a listing for patients with ALF include (1) care in an intensive care unit, (2) renal replacement therapy, (3) ventilator dependence, and (4) INR $\geq 2$ with development of HE within 8 weeks of the initial liver injury [3••].

The decision to proceed to transplant and the timing of surgery are of critical consequence. A patient who might have spontaneously recovered with supportive care will be subjected to a high-risk surgery and lifelong immunosuppression if LT is pursued too aggressively. At the same time, observing an ALF patient for too long may lead to multiorgan dysfunction that ultimately precludes transplant. Data suggest that the etiology of ALF may help predict recovery with supportive care. For example, patients with acetaminophen, ischemia, or hepatitis A-induced ALF are more likely to have spontaneous recovery ( $\sim 60 \%)$ as compared with ALF incited by autoimmune hepatitis, hepatitis B, idiosyncratic drug reactions, Wilson's disease, or Budd-Chiari ( 25\%) [5]. Several prognostic models have also been developed to assist with transplant decision-making. The most widely used is the King's College Criteria [7]. Data from 588 patients with ALF who received medical management were used to construct prognostic scoring systems for acetaminophen-induced and non-acetaminophen-induced ALF. Numerous studies have evaluated the clinical performance of the criteria, with sensitivity and specificity estimates for predicting mortality ranging from 58 to $68 \%$ and $82-95 \%$, respectively [8]. Other predictive scoring systems used in ALF include the Clichy criteria, MELD, and the Acute Liver Failure Study Group (ALFSG) index.

\section{Decompensated or Advanced Cirrhosis}

Cirrhosis affects more than 600,000 individuals in the US and decreases survival relative to the general population [9]. Cirrhosis alone, however, is not an indication for LT. Based on the risk of transplant and scarcity of donor organs, most patients with cirrhosis are considered too healthy for transplant. At the same time, end-stage liver disease causes an estimated 50,000 to 75,000 deaths in the US annually [10]. Fewer than $20 \%$ of these patients are referred for transplant evaluation, and fewer still are ultimately listed for transplant.
Between the extremes of relative health and critical illness lie the criteria for transplant evaluation in patients with cirrhosis. It is generally accepted that LT may be appropriate in patients with (1) decompensated cirrhosis or (2) MELD $\geq 15$.

The primary decompensations of cirrhosis are ascites, variceal hemorrhage, HE, and jaundice. The transition from compensated to decompensated disease is a seminal event in the natural history of cirrhosis, and occurs at a rate of 5-7\% per year. Decompensation reduces median survival from $>$ 12 years to $\sim 2$ years [11], and accumulation of multiple decompensations further decreases survival. Given these steep increments in mortality, index decompensation should prompt referral for transplant evaluation. There is nuance to this general rule, however, as certain conditions may cause decompensations that are potentially reversible. For example, patients with alcoholic hepatitis often develop ascites, encephalopathy, and jaundice, but with supportive care and alcohol abstinence, patients may return to a compensated state. Similarly, flares of autoimmune hepatitis may respond briskly to immunosuppression and patients with unchecked hepatitis $\mathrm{B}$ may respond to antivirals. In these limited circumstances, decompensation may be reversed with appropriate therapies, and LT evaluation may not be necessary. Of course, it can be difficult to discern if a given patient will experience this degree of improvement, and transplant evaluation should be actively considered based on the clinical course.

The second broad indication for consideration of LT in cirrhosis is a MELD $\geq 15$. In a landmark study by Merion et al., wait list and post-transplant mortality were followed in a cohort of 12,996 patients with cirrhosis. The HR (HR) for 1year post-transplant mortality was higher than waiting list mortality in patients with a MELD < 15 (HR 1.76 for MELD 12-14, $p=0.04$ ) [12]. In essence, a survival inflection point exists in the MELD spectrum where the associated risks of transplant outweigh the benefits. In practice, many physicians refer patients for transplant referral once the MELD score exceeds 10 . This allows the patient to initiate the evaluation process, address any potential barriers to transplantation, and become familiar with the transplant team. The patient can thus be listed more expeditiously once the MELD score reaches 15 or more.

\section{Primary Hepatic Neoplasms}

The two primary hepatic malignancies that may potentially be treated with LT are hepatocellular carcinoma (HCC) and hilar cholangiocarcinoma. HCC is an aggressive hepatic cancer that develops in patients with cirrhosis or chronic liver disease. It has a high propensity for multifocal spread, which in many cases limits the role of surgical resection. LT has thus been an attractive option for treatment as it may cure HCC while also addressing underlying chronic liver disease. Early experience with LT for unselected HCC patients was characterized by 
frequent tumor recurrence and poor outcomes [13]. However, with the recognition that patients with smaller HCC foci fared better with LT, specific criteria were established to assist with appropriate patient selection. These so-called Milan criteria were defined in a landmark study by Mazzaferro et al., and require a single tumor $\leq 5 \mathrm{~cm}$ or no more than three tumors each $\leq 3 \mathrm{~cm}$. Four-year survival for patients with Milan criteria was $75 \%$, similar to cirrhotic patients without HCC undergoing transplant [14]. UNOS currently uses the Milan criteria to assign MELD exception points to patients with HCC, who often have low MELD scores that do not adequately reflect short-term mortality [15]. Patients who are outside of the Milan criteria may be downstaged with therapies such as radiofrequency ablation or transarterial chemoembolization, although the process for assigning exception points in these cases is evolving.

Cholangiocarcinoma is a rare but extremely aggressive malignancy arising from the biliary tree, most commonly encountered in patients with primary sclerosing cholangitis. Untreated cholangiocarcinoma has a $9 \%$ survival rate at 3 years [16]. Although surgical resection is the only viable treatment option for patients with intrahepatic or extrahepatic cholangiocarcinoma, highly selected patients with hilar malignancy (i.e., tumor arising near the hepatic duct bifurcation) may benefit from LT. Resection is frequently contraindicated for hilar lesions because of bilateral liver involvement, encasement of vascular structures, or local lymphatic invasion. Initial attempts to transplant such patients were met with poor outcomes, with $<30 \% 5$-year survival [17]. Subsequently, the Mayo Clinic and University of Nebraska pioneered a protocol introducing neoadjuvant radiochemotherapy prior to LT in patients with hilar cholangiocarcinoma. This yielded 5-year survival rates in excess of $80 \%$ [18]. As such, in 2009, UNOS began granting MELD exception points to patients with unresectable, non-metastatic hilar cholangiocarcinoma who have received an approved neoadjuvant therapy protocol. The benefit of this transplant approach was confirmed in a 2012 study of 12 transplant centers, where the recurrencefree survival at 5 years was found to be $65 \%$ among 214 transplanted patients [19].

\section{Inborn Metabolic Conditions}

Several genetic disorders that cause hepatic injury are eligible for MELD exception points, including cystic fibrosis (CF), primary hyperoxaluria, and familial amyloid polyneuropathy (FAP). CF is an autosomal recessive disorder that impairs the functionality of the CFTR (CF transmembrane conductance regulator) chloride channel, resulting in thickened exocrine gland secretions that lead to multiorgan dysfunction such as obstructive airway disease and pancreatic insufficiency. Hepatobiliary disease is characterized by inspissation of the biliary tree, progressive cholestasis, and occasionally cirrhosis with portal hypertension. Because CF patients with lung and liver disease have increased mortality with lung transplant alone, combined lung-liver transplantation may be indicated. Currently, for MELD exception points, the patient must have a forced expiratory volume at $1 \mathrm{~s}$ (FEV1) of less than 0.40 and a CF diagnosis confirmed by genetic testing [20].

Primary hyperoxaluria type I is an autosomal recessive alanine:glyoxylate aminotransferase (AGT) enzyme defect that results in hyperoxaluria, oxylate stone formation, and progressive renal failure. Kidney transplantation alone will not correct the underlying enzyme deficiency, as ATG is normally present in the liver. Consequently, combined liverkidney transplant is the only definitive cure. Exception criteria include significant renal insufficiency (glomerular filtration rate $\leq 25 \mathrm{~mL} / \mathrm{min}$ or on dialysis), dual listing for liverkidney transplant, and AGT deficiency as confirmed on liver biopsy or genetic testing [20].

Finally, FAP is an autosomal dominant hereditary amyloidosis caused by a mutation in the transthyretin (TTR) gene. Although many variants exist, the most common is a Val30Met point mutation, occurring in $\sim 85 \%$ of FAP patients. TTR mutation results in widespread progressive amyloid deposition, principally involving the liver, heart, kidneys, GI tract, eyes, and the peripheral and autonomic nervous system. Because TTR is almost exclusively produced in the liver, transplantation may restore normal TTR production and be lifesaving. However, it has been demonstrated that ocular and cardiac disease can progress despite LT, and as such, younger patients with a shorter duration of disease may have better post-transplant outcomes [21]. Exception criteria include ability to ambulate independently, cardiac ejection fraction $>40 \%$ or dual heart-liver transplant listing, biopsyproven amyloid organ involvement, and identification of the TTR mutation [20].

\section{Contraindications to Liver Transplantation}

\section{Absolute Contraindications}

An important step in the evaluation process is recognizing which patients should not be transplanted. Although contraindications vary considerably by transplant center, there are a number of accepted absolute contraindications. These include severe cardiopulmonary disease, uncontrolled sepsis, active extrahepatic malignancy, acquired immune deficiency syndrome (AIDS), brain death, and anatomic or technical barriers to transplant (Table 1). Active alcohol or illicit substance abuse is an additional absolute contraindication, but the duration of abstinence required prior to listing is variable. Many institutions require at least 6 months of demonstrated abstinence and enrollment in a substance abuse program. This standard is based literature that sobriety less than 6 months 
is an independent predictor of post-LT recidivism [22], although the data are controversial [23]. As such, many transplant centers are more lenient with regard to abstinence duration.

\section{Relative Contraindications}

Relative contraindications are institution and patientdependent (Table 1). First, advanced age, often over 65 years, is a relative contraindication. Early data suggested that older age was associated with poor graft function and decreased survival [24]; however, more recent data showed that appropriately selected transplant recipients over 65 had equivalent 5 -year survival outcomes as compared with younger cohorts [25]. Furthermore, older patients had fewer episodes of acute rejection, possibly related to relative immune senescence. AASLD guidelines argue that physiological age is more important than chronological age, and even patients over 70 may be appropriate for transplant if the comorbidity assessment is favorable [3••]. Second, extensive portal vein thrombosis complicates the technical aspects of LT, and may be a relative contraindication. Because the liver allograft must receive adequate portal flow, the transplant risk of portal venous thrombosis requires an assessment of clot burden and degree of collateralization. Extension of thrombosis into the splanchnic vasculature heralds a particularly high transplant mortality risk [26]. Third, medical non-compliance and poor psychosocial circumstances may increase the risk of non-adherence to immunosuppression and routine post-transplant care. Efforts should be made to improve support systems as able, but if refractory, these factors may preclude transplant. Finally, based on data summarized previously, a MELD score < 15 signifies an unfavorable risk/benefit analysis for most patients and is a relative contraindication to transplant.

Other important relative contraindications include human immunodeficiency virus (HIV) positivity, cholangiocarcinoma, and obesity. HIV-positive patients exist along a spectrum of risk for transplant. Those with extremely low CD4 counts or an AIDS-defining illness are typically considered to have an absolute contraindication; however, patients with wellcontrolled HIV on antiretroviral therapy may do well posttransplant. Per AASLD recommendations, a patient should have a CD4 count $>100 / \mu \mathrm{L}$ and an undetectable viral load at the time of transplant [3*0]. Early experience with transplantation of HIV-positive patients showed adequate short-term survival outcomes, although patients with hepatitis $\mathrm{C}$ virus $(\mathrm{HCV})$ coinfection had higher mortality rates [27]. Patients with HIV$\mathrm{HCV}$ coinfection experience more severe $\mathrm{HCV}$ recurrence post-transplant and poorer survival than those with $\mathrm{HCV}$ alone [28]; however, in the modern era of highly effective directacting antivirals for treating $\mathrm{HCV}$, the transplant outcomes of HIV-HCV coinfected patients are likely to improve.
Cholangiocarcinoma was previously regarded to be an absolute contraindication to transplant. However, patients with hilar cholangiocarcinoma may be candidates for LT provided a specific protocol of neoadjuvant radiochemotherapy is followed prior to transplant. Intrahepatic and extrahepatic cholangiocarcinoma remain absolute contraindications to LT. Finally, morbid obesity (body mass index $[\mathrm{BMI}] \geq 40$ ) is a relative contraindication to transplant, as this confers significantly higher mortality, increased risk of primary graft non-function, prolonged length of hospital stay, and higher cost of transplant [29]. Attempts at weight loss should be pursued prior to transplant, although it is important to note that bariatric surgery is contraindicated in decompensated cirrhosis.

\section{Evaluation for Liver Transplantation}

LT entails the physical and hemodynamic demands of a major surgery, a potentially protracted recovery period, the risks of chronic immunosuppression, and increased psychosocial stress. As such, the pre-transplant evaluation process is designed to screen for patients who (1) are physically healthy enough to survive the operative and perioperative periods, (2) are adherent with medical recommendations to ensure compliance with postoperative care, and (3) have a secure psychosocial support system. Broadly speaking, the transplant evaluation involves expert consultation, basic laboratory analysis, cardiopulmonary testing, malignancy screening, and a psychosocial assessment (Table 2). As with most aspects of transplant, the specific battery of testing varies by institution.

\section{Expert Consultation}

The candidate must meet a hepatologist and a transplant surgeon to confirm candidacy for transplant by reviewing the history of liver disease, including the duration, severity, and prognosis without transplant. It should be confirmed that the limits of medical therapy have been reached, and comorbidities that might preclude or otherwise limit the benefit of transplantation should be identified. The hepatologist should ensure that general cirrhosis care has been performed, including esophageal variceal screening and therapy, imaging for hepatocellular carcinoma, and vaccination for hepatitis A and B, among other measures. The transplant surgeon should evaluate for anatomic/technical barriers to surgery, and assist with identifying a donor and graft approach that reflects the patient's circumstances. In all cases, these interactions offer a critical opportunity for the patient and family to receive education about the transplant process and have all questions answered. 
Table 2 Overview of testing and evaluation prior to liver transplantation

\begin{tabular}{|c|c|}
\hline Item & Detail \\
\hline Expert consultation & $\begin{array}{l}\text { Meeting with transplant hepatologist and transplant surgeon to review appropriateness } \\
\text { of transplant evaluation and address questions/concerns. }\end{array}$ \\
\hline Basic laboratory testing & $\begin{array}{l}\text { Blood typing, comprehensive metabolic panel, complete blood counts, coagulation } \\
\text { parameters, urinalysis, drug screen, calcium, and vitamin D. }\end{array}$ \\
\hline Infectious disease testing & $\begin{array}{l}\text { Viral hepatitis serologies, tuberculosis testing, and RPR for syphilis. Consideration } \\
\text { of coccidioides and strongyloides testing if indicated. }\end{array}$ \\
\hline Cardiac evaluation & $\begin{array}{l}\text { Electrocardiography and echocardiography. Non-invasive cardiac stress testing, } \\
\text { with cardiac catheterization if abnormal. }\end{array}$ \\
\hline Pulmonary evaluation & $\begin{array}{l}\text { Pulse oximetry. If }<96 \% \text { on room air, perform arterial blood gas sampling. Bubble } \\
\text { echocardiography, pulmonary function tests and chest } \mathrm{CT} \text { if concern for arterial } \\
\text { hypoxemia. Routine echocardiography with right heart catheterization if concern } \\
\text { for pulmonary arterial hypertension. }\end{array}$ \\
\hline Age-appropriate malignancy screening & $\begin{array}{l}\text { Colonoscopy, mammography, Papanicolaou screening, and low-dose chest } \mathrm{CT} \text { as indicated. } \\
\text { Hepatocellular carcinoma screening with abdominal imaging } \pm \text { alpha fetoprotein testing. }\end{array}$ \\
\hline Hepatic imaging & Triple-phase CT, gadolinium-enhanced MRI, or abdominal ultrasound with doppler imaging. \\
\hline Bone density testing & Calcium, vitamin $\mathrm{D}$, and dual-energy $\mathrm{X}$-ray absorptiometry. \\
\hline Psychiatric evaluation & Address substance abuse and active psychiatric conditions, as indicated. \\
\hline Social work evaluation & Identify psychosocial barriers to transplantation and initiate remediation. \\
\hline Nutritional evaluation & Assess nutritional status and implement plan to address BMI extremes. \\
\hline Financial and insurance screening & Ensure adequate coverage for pre-transplant evaluation, transplantation, and post-transplant care. \\
\hline
\end{tabular}

Adapted from the 2013 AASLD guidelines [3••]

\section{Laboratory Testing}

The main purpose of pre-LT laboratory testing is to confirm the blood type (which must match a donor organ), assess the state of hepatic and renal function, and rule out concomitant infections that might require treatment or otherwise impact post-transplant management and outcomes. This includes:

- $\mathrm{ABO}$ and $\mathrm{Rh}$ blood typing

- Basic metabolic panel

- Complete blood count including differential

- Liver-associated enzymes and coagulation parameters

- Calcium and vitamin D

- Urinalysis

- Urine drug screen

- Serologies for hepatitis A, hepatitis B, hepatitis C, HIV, varicella zoster virus (VZV), cytomegalovirus (CMV), Epstein-Barr virus (EBV), and rapid plasma reagin (RPR)

- Latent tuberculosis screening: interferon- $\gamma$ release assay or tuberculin skin testing

\section{Cardiac Evaluation}

The goal of cardiac testing prior to LT is to detect significant coronary artery disease (CAD), valvular pathology, and/or cardiomyopathy. If present, specific interventions may be pursued prior to or during transplant, as indicated. If there is advanced cardiac pathology, transplant may be contraindicated. Estimates of CAD prevalence in cirrhosis range from 2.5 to $28 \%$, as compared with 2.5 to $6.9 \%$ in the general population [30]. The prevalence of CAD increases with age, and $\mathrm{LT}$ recipients with $\mathrm{CAD}$ have higher morbidity and mortality as compared with those without CAD [31].

The optimal approach to cardiac testing has not been defined. Electrocardiography and echocardiography should be performed in all patients considered for transplant. This will allow for detection of arrhythmias, conduction abnormalities, valvular disease, and ventricular dysfunction. To evaluate for CAD, the AASLD practice guidelines recommend that all LT candidates undergo non-invasive stress testing $[3 \bullet \bullet$. Because many patients with advanced liver disease are unable to reach the minimum heart rate parameters for an exercise stress test, pharmacological stress tests are typically performed, with dobutamine stress tests being the most common. The American Heart Association and American College of Cardiology Foundation, in contrast, recommends non-invasive cardiac stress testing only in patients with three or more risk factors for CAD [32]. These include diabetes, hypertension, hyperlipidemia, smoking, age greater than 60, known cardiovascular disease, and left ventricular hypertrophy.

If non-invasive stress testing is abnormal, cardiac catheterization should be performed. If significant CAD exists $(>70 \%$ stenosis), revascularization should be considered prior to LT. Because of the risks of major cardiac surgery in decompensated cirrhosis, revascularization through percutaneous 
coronary intervention is preferred, if feasible. Bare metal stent placement has the advantage of avoiding prolonged dual antiplatelet therapy (DAPT), which may delay LT. Importantly, there is recent data demonstrating that DAPT in the setting of stenting for $\mathrm{CAD}$ increases the risk of gastrointestinal bleeding in cirrhotics but does not adversely impact survival [33•]. As such, DAPT should not be avoided or withheld simply based on the presence of cirrhosis.

\section{Pulmonary Evaluation}

The major pulmonary complications of end-stage liver disease are hepatopulmonary syndrome (HPS) and portopulmonary hypertension (PPHTN). HPS is defined by the triad of arterial hypoxemia, intrapulmonary vascular dilation, and chronic liver disease. Although the pathophysiology is poorly defined, vasoactive mediators such as nitric oxide produced in response to portal hypertension may lead to angiogenesis and pulmonary vasodilation. This results in widespread intrapulmonary shunting at the capillary and pre-capillary level with resultant arterial hypoxemia. HPS develops in 5 to $32 \%$ of cirrhotics [3*0] and significantly increases the risk of death in patients undergoing transplant evaluation (HR 2.41, $p=0.005$ ) [34]. Pulse oximetry is thus indicated in all LT patients to screen for this condition. If the oxygen saturation is $<96 \%$ and the patient has symptoms that could be explained by HPS (i.e., progressive dyspnea, platypnea, orthodeoxia), additional diagnostics are warranted. Arterial blood gas sampling will provide the arterial oxygen tension $\left(\mathrm{P}_{\mathrm{a}} \mathrm{O}_{2}\right)$ and contrast/bubble echocardiography can identify intrapulmonary shunting. Because the short-term mortality of cirrhosis with HPS is poorly characterized by MELD, exception points may be obtained if the following criteria are met: $\mathrm{P}_{\mathrm{a}} \mathrm{O}_{2}<60 \mathrm{mmHg}$, evidence of intrapulmonary shunting, and portal hypertension [20]. Importantly, alternative explanations of arterial hypoxemia must be ruled out, typically with pulmonary function testing and chest CT. After LT, patients with HPS experience a clear survival benefit and in those who survive to at least 6 months HPS frequently resolves [35].

PPHTN refers to unexplained pulmonary arterial hypertension (PAH) that occurs in the context of portal hypertension. Clinically significant PPHTN occurs in up to $5 \%$ of cirrhotics referred for LT [36], and may cause progressive dyspnea, fatigue, and right-sided heart failure. Although the pathophysiology is unknown, humoral factors normally processed in the liver may reach the pulmonary system via portosystemic shunting and induce portal hypertension. Patients with PPHTN undergoing LT have significantly increased shortterm mortality. Krowka et al. showed that in patients with mean pulmonary arterial pressure (MPAP) from 35 to $50 \mathrm{mmHg}$, the perioperative mortality was $50 \%$, and in those with MPAP $>50 \mathrm{mmHg}$, the mortality was $100 \%$ [37]. Routine echocardiography can be used to estimate the pulmonary arterial systolic pressure (PASP) and, if contrast enhanced, the right ventricular systolic pressure (RVSP). PAH is possible if the PASP is $36-49 \mathrm{mmHg}$, and likely if > $50 \mathrm{mmHg}$. A RVSP greater than or equal to $45 \mathrm{mmHg}$ also suggests PAH. If any of these findings are present, right heart catheterization should be performed. The diagnosis of PAH is confirmed upon findings of MPAP $\geq 25 \mathrm{mmHg}$ and a pulmonary capillary wedge pressure $\leq 15 \mathrm{mmHg}$. A pulmonary vascular resistance $(\mathrm{PVR}) \geq 240 \mathrm{dyne} / \mathrm{s} / \mathrm{cm}^{-5}$ is also consistent with the diagnosis. Although mild PAH (MPAP < $35 \mathrm{mmHg}$ ) does not appear to impact LT outcomes, patients with more severe PAH should be referred to a PAH specialist for consideration of vasodilator therapies. If a patient on therapy can achieve an MPAP $<35 \mathrm{mmHg}$ and PVR $<400$ dyne $/ \mathrm{s} / \mathrm{cm}^{-5}$, LT can proceed with reasonable short-term outcomes [3••]. Similar to HPS, MELD exception points may be obtained if the patient meets the above criteria on treatment for PPHTN (MPAP $<35 \mathrm{mmHg}$ and PVR $<400$ dyne $/ \mathrm{s} / \mathrm{cm}^{-5}$ ) and has a hepatic venous pressure gradient $\geq 12 \mathrm{mmHg}$ [20].

\section{Malignancy Screening}

Because the presence of extrahepatic malignancy is an absolute contraindication to LT, age-appropriate cancer screening should be performed in all patients. The specific workup depends on the demographics and risk factors of the patient, and should be performed in accordance with guideline-based recommendations, such as the US Preventive Services Task Force. Testing may include colonoscopy to rule out colorectal cancer, mammography to rule our breast cancer, Papanicolaou smear to rule our cervical cancer, and low-dose chest computed tomography (CT) screening to rule out lung cancer. Additionally, it should be ensured that the patient is up-todate on hepatocellular cancer screening, which may be performed with an abdominal ultrasound, spiral CT scan, or magnetic resonance imaging (MRI), with or without alphafetoprotein sampling. In patients with an incident malignancy or a prior history of malignancy, the pursuit of curative treatment and demonstration of tumor-free survival are requisite for LT candidacy.

\section{Infectious Disease Considerations}

The main infectious disease priorities prior to transplant are to detect active infections, treat them as indicated, and to ensure appropriate preventative vaccination. Laboratory screening should include hepatitis $\mathrm{A} / \mathrm{B} / \mathrm{C}$ serologies, CMV, EBV, $\mathrm{VZV}$, and RPR. Testing for tuberculosis (TB) may be completed with a tuberculin skin test or an interferon-gamma release assay (i.e., Quantiferon or T.SPOT). If the patient has resided in an area endemic to Coccidioides (southwestern US) or Strongyloides (portions of southeastern US), appropriate serologic testing should be obtained. A dental examination 
should also be completed and any necessary extractions performed prior to transplant.

Latent tuberculosis should be treated prior to transplantation if possible. This can be accomplished with isoniazid- or rifampin-based therapies, both of which are safe to use in advanced liver disease with proper monitoring. Syphilis should also be treated prior to transplantation, typically with penicillin. If coccidioides serologies are positive, indefinite fluconazole prophylaxis should be considered post-transplant. The patient must also be ruled out for active coccidioidomycosis. If present, treatment of active disease requires confirmation of clearance and resolution of symptoms, a process that may delay transplant candidacy by 12 months or more. Finally, positive strongyloides serologies should prompt treatment with ivermectin prior to transplant.

Patients should be vaccinated against hepatitis A and B if serologies do not demonstrate immunity. Tetanus, diphtheria, and pertussis vaccinations should be up-to-date, as well as immunization against influenza and pneumococcus, which includes both 23-valent and 13-valent preparations. If indicated, human papilloma virus vaccination should be completed. Finally, if live vaccinations are required (i.e., measles, mumps, rubella, VZV), these should be performed as early as possible in the evaluation process, as live vaccination is contraindicated after transplant, and a minimum of 4 weeks should separate live vaccination and organ transplantation.

\section{Hepatic Imaging}

Cross-sectional imaging sufficient for surgical planning should be obtained if not already performed. Triple-phase CT or gadolinium-enhanced MRI is most common, and may help identify potential technical barriers to transplant, including portal venous thrombosis, perihepatic varices, anatomic anomalies, and sequelae of prior abdominal surgeries. If CT or MRI cannot be obtained, ultrasound with assessment of Doppler flows may suffice. As reviewed elsewhere, hepatic imaging is particularly critical to accurately diagnose and stage patients with intrahepatic malignancy in order to confirm transplant candidacy (i.e., Milan criteria for HCC and hilar location for cholangiocarcinoma).

\section{Bone Density Testing}

Advanced liver disease promotes osteoporosis through relative inactivity, hypogonadism, reduced vitamin D levels, impaired intestinal calcium absorption, and, in certain patients, the direct effects of alcohol, steroids, or iron overload. The prevalence of osteoporosis in cirrhosis ranges from 15 to $56 \%$ [38]. Patients with cholestatic liver disease such as primary biliary cirrhosis are at particularly high risk. After transplant, several months of obligate high-dose steroids further reduce bone mineral density. Indeed, studies demonstrate a 1-year post-transplant fracture risk in the 30th percentile [39]. As such, vitamin $\mathrm{D}$ and calcium testing in addition to dualenergy X-ray absorptiometry is an essential part of the transplant evaluation. If osteopenia or osteoporosis is detected, appropriate treatment should be initiated prior to transplant in order to optimize bone density and minimize fracture risk. This includes vitamin D and calcium supplementation for all patients. Bisphosphonate therapy, when indicated, may also improve bone density and reduce fracture risk in the transplant setting [40].

\section{Other Assessments}

A formal psychosocial evaluation with a social worker and, if indicated, a psychiatrist is necessary prior to transplant. The purpose of this evaluation is to identify potential psychosocial contraindications to transplant and initiate appropriate interventions as early as possible. As detailed previously, these concerns include active alcohol or substance abuse, poor medical compliance, and inadequate social support. Nutritional status should also be assessed to identify patients at the extremes of BMI. The risks of morbid obesity (BMI $\geq 40$ ) were discussed above, but there is also clear evidence that underweight patients $(\mathrm{BMI}<18.5)$ have higher wait list mortality and worse LT outcomes as compared with those with normal BMI [41]. These patients require consultation with a dietician and probable initiation of enteral or parenteral feeding prior to transplantation. Finally, financial and insurance screening must be performed to ensure adequate coverage for the evaluation, transplant, and post-transplant phases of care.

\section{Conclusion}

LT is an important therapeutic option for patients with lifelimiting liver disease. Transplant evaluation involves extensive testing to ensure that the patient can withstand the demands of transplant surgery and post-transplant care. Ongoing organ supply/demand mismatch further dictates the need for strict indications and contraindications in identifying LT-suitable candidates. This ultimately shapes the specific criteria for patient selection, which will continue to evolve as means are developed to expand the donor pool, improve surgical techniques, broaden indications for safe transplant, and extend the lifetime of a graft. It is therefore critical for physicians to remain familiar with the general indications for transplantation and to know when it is appropriate or inappropriate to refer for transplant evaluation.

\section{Compliance with Ethical Standards}

Conflict of Interest Dr. Mahmud declares no conflicts of interest related to the current work. 
Human and Animal Rights and Informed Consent This article does not contain any studies with human or animal subjects performed by any of the authors.

\section{References}

Papers of particular interest, published recently, have been

highlighted as:

- Of importance

•- Of major importance

1. Duffy JP, Kao K, Ko CY, Farmer DG, McDiarmid SV, Hong JC, et al. Long-term patient outcome and quality of life after liver transplantation: analysis of 20-year survivors. Ann Surg. 2010;252(4): 652-61.

2.• Organ Procurement and Transplantation Network: National Data for liver transplantation. 2017. Available from: https://optn. transplant.hrsa.gov/data/view-data-reports/national-data/\#. Accessed 5 Mar 2020. National liver transplantation statistics are publicly available at the OPTN website.

3.•• Martin P, DiMartini A, Feng S, Brown R, Fallon M. Evaluation for liver transplantation in adults: 2013 practice guideline by the American Association for the Study of Liver Diseases and the American Society of Transplantation. Hepatology. 2014;59(3): 1144-65 These are the national society guidelines regarding the indications and contraindications for liver transplantation, as well as an overview of the transplant evaluation process.

4. Lee WM. Acute liver failure. N Engl J Med. 1993;329(25):186272.

5. Ostapowicz G, Fontana RJ, Schiødt FV, Larson A, Davern TJ, Han $\mathrm{SH}$, et al. Results of a prospective study of acute liver failure at 17 tertiary care centers in the United States. Ann Intern Med. 2002;137(12):947-54.

6. Larson AM, Polson J, Fontana RJ, Davern TJ, Lalani E, Hynan LS, et al. Acetaminophen-induced acute liver failure: results of a United States multicenter, prospective study. Hepatology. 2005;42(6): 1364-72.

7. O'Grady JG, Alexander GJ, Hayllar KM, Williams R. Early indicators of prognosis in fulminant hepatic failure. Gastroenterology. 1989;97(2):439-45.

8. Craig DG, Ford AC, Hayes PC, Simpson KJ. Systematic review: prognostic tests of paracetamol-induced acute liver failure. Aliment Pharmacol Ther. 2010;31(10):1064-76.

9. Scaglione S, Kliethermes S, Cao G, Shoham D, Durazo R, Luke A, et al. The epidemiology of cirrhosis in the United States: a population-based study. J Clin Gastroenterol. 2015;49(8):690-6.

10. Merion RM, editor Current status and future of liver transplantation. Seminars in liver disease; 2010: (C) Thieme Medical Publishers.

11. D'Amico G, Garcia-Tsao G, Pagliaro L. Natural history and prognostic indicators of survival in cirrhosis: a systematic review of 118 studies. J Hepatol. 2006;44(1):217-31.

12. Merion RM, Schaubel DE, Dykstra DM, Freeman RB, Port FK, Wolfe RA. The survival benefit of liver transplantation. Am J Transplant. 2005;5(2):307-13.

13. Pichlmayr R, Weimann A, Ringe B. Indications for liver transplantation in hepatobiliary malignancy. Hepatology. 1994;20(S7):S3340.

14. Mazzaferro V, Regalia E, Doci R, Andreola S, Pulvirenti A, Bozzetti F, et al. Liver transplantation for the treatment of small hepatocellular carcinomas in patients with cirrhosis. N Engl J Med. 1996;334(11):693-700.
15. Ioannou GN, Perkins JD, Carithers RL. Liver transplantation for hepatocellular carcinoma: impact of the MELD allocation system and predictors of survival. Gastroenterology. 2008;134(5):134251.

16. Farley DR, Weaver AL, Nagorney DM, editors. "Natural history" of unresected cholangiocarcinoma: patient outcome after noncurative intervention. Mayo Clinic Proceedings; 1995: Elsevier.

17. Robles R, Figueras J, Turrión VS, Margarit C, Moya A, Varo E, et al. Spanish experience in liver transplantation for hilar and peripheral cholangiocarcinoma. Ann Surg. 2004;239(2):265-71.

18. Rosen CB, Heimbach JK, Gores GJ. Liver transplantation for cholangiocarcinoma. Transpl Int. 2010;23(7):692-7.

19. Murad SD, Kim WR, Harnois DM, Douglas DD, Burton J, Kulik LM, et al. Efficacy of neoadjuvant chemoradiation, followed by liver transplantation, for perihilar cholangiocarcinoma at 12 US centers. Gastroenterology. 2012;143(1):88-98. e3.

20. (UNOS) UNfOS. Submitting standard MELD/PELD exception scores 2010 [Available from: https://www.transplantpro.org/news/ submitting-standardized-meldpeld-exception-scores/. Accessed 5 Mar 2020.

21. Moini M, Mistry P, Schilsky ML. Liver transplantation for inherited metabolic disorders of the liver. Curr Opin Organ Transplant. 2010;15(3):269-76.

22. Osorio RW, Ascher NL, Avery M, Bacchetti P, Roberts JP, Lake JR. Predicting recidivism after orthotopic liver transplantation for alcoholic liver disease. Hepatology. 1994;20(1):105-10.

23. Foster PF, Fabrega F, Karademir S, Sankary HN, Mital D, Williams JW. Prediction of abstinence from ethanol in alcoholic recipients following liver transplantation. Hepatology. 1997;25(6):1469-77.

24. Ploeg RJ, D'alessandro AM, Knechtle SJ, Stegall MD, Pirsch JD, Hoffmann RM, et al. Risk factors for primary dysfunction after liver transplantation-a multivariate analysis. Transplantation. 1993;55(4):807-13.

25. Cross TJ, Antoniades CG, Muiesan P, Al-Chalabi T, Aluvihare V, Agarwal K, et al. Liver transplantation in patients over 60 and 65 years: an evaluation of long-term outcomes and survival. Liver Transpl. 2007;13(10):1382-8.

26. Manzanet G, Sanjuán F, Orbis P, López R, Moya A, Juan M, et al. Liver transplantation in patients with portal vein thrombosis. Liver Transpl. 2001;7(2):125-31.

27. Radecke K, Frühauf NR, Miller M, Ross B, Köditz R, Malagó M, et al. Outcome after orthotopic liver transplantation in five HIVinfected patients with virus hepatitis-induced cirrhosis. Liver Int. 2005;25(1):101-8.

28. Duclos-Vallée JC, Féray C, Sebagh M, Teicher E, Roque-Afonso AM, Roche B, et al. Survival and recurrence of hepatitis $\mathrm{C}$ after liver transplantation in patients coinfected with human immunodeficiency virus and hepatitis $C$ virus. Hepatology. 2008;47(2):407-17.

29. Nair S, Verma S, Thuluvath PJ. Obesity and its effect on survival in patients undergoing orthotopic liver transplantation in the United States. Hepatology. 2002;35(1):105-9.

30. Plotkin JS, Benitez RM, Kuo PC, Njoku MJ, Ridge LA, Lim JW, et al. Dobutamine stress echocardiography for preoperative cardiac risk stratification in patients undergoing orthotopic liver transplantation. Liver Transpl. 1998;4(4):253-7.

31. Plotkin JS, Scott VL, Pinna A, Dobsch BP, De Wolf AM, Kang Y. Morbidity and mortality in patients with coronary artery disease undergoing orthotopic liver transplantation. Liver Transpl. 1996;2(6):426-30.

32. Lentine KL, Costa SP, Weir MR, Robb JF, Fleisher LA, Kasiske $\mathrm{BL}$, et al. Cardiac disease evaluation and management among kidney and liver transplantation candidates: a scientific statement from the American Heart Association and the American College of Cardiology Foundation. J Am Coll Cardiol. 2012;60(5):434-80.

33. Krill T, Brown G, Weideman R, Cipher D, Spechler S, Brilakis E, et al. Patients with cirrhosis who have coronary artery disease 
treated with cardiac stents have high rates of gastrointestinal bleeding, but no increased mortality. Aliment Pharmacol Ther. 2017;46(2):183-92. Patients with cirrhosis and coronary artery disease who receive stenting and dual antiplatelet therapy have an increased risk of gastrointestinal bleeding, but no adverse impact on survival. This may impact decision-making surrounding revascularization of coronary artery disease in the peri-transplant setting.

34. Fallon MB, Krowka MJ, Brown RS, Trotter JF, Zacks S, Roberts $\mathrm{KE}$, et al. Impact of hepatopulmonary syndrome on quality of life and survival in liver transplant candidates. Gastroenterology. 2008;135(4):1168-75.

35. Arguedas MR, Abrams GA, Krowka MJ, Fallon MB. Prospective evaluation of outcomes and predictors of mortality in patients with hepatopulmonary syndrome undergoing liver transplantation. Hepatology. 2003;37(1):192-7.

36. Colle IO, Moreau R, Godinho E, Belghiti J, Ettori F, Cohen-Solal A, et al. Diagnosis of portopulmonary hypertension in candidates for liver transplantation: a prospective study. Hepatology. 2003;37(2):401-9.

37. Krowka MJ, Plevak DJ, Findlay JY, Rosen CB, Wiesner RH, Krom RA. Pulmonary hemodynamics and perioperative cardiopulmonary-related mortality in patients with portopulmonary hypertension undergoing liver transplantation. Liver Transpl. 2000;6(4):443-50.

38. Ninkovic M, Love S, Tom B, Alexander G, Compston J. High prevalence of osteoporosis in patients with chronic liver disease prior to liver transplantation. Calcif Tissue Int. 2001;69(6):321-6.

39. Leidig-Bruckner G, Hosch S, Dodidou P, Ritschel D, Conradt C, Klose C, et al. Frequency and predictors of osteoporotic fractures after cardiac or liver transplantation: a follow-up study. Lancet. 2001;357(9253):342-7.

40. Guañabens N, Monegal A, Cerdá D, Muxí Á, Gifre L, Peris P, et al. Randomized trial comparing monthly ibandronate and weekly alendronate for osteoporosis in patients with primary biliary cirrhosis. Hepatology. 2013;58(6):2070-8.

41. Dick AA, Spitzer AL, Seifert CF, Deckert A, Carithers RL, Reyes $\mathrm{JD}$, et al. Liver transplantation at the extremes of the body mass index. Liver Transpl. 2009;15(8):968-77.

Publisher's Note Springer Nature remains neutral with regard to jurisdictional claims in published maps and institutional affiliations. 